\title{
DIMENSIONS OF JULIA SETS OF MEROMORPHIC FUNCTIONS
}

\author{
P. J. RIPPON AND G. M. STALLARD
}

\begin{abstract}
It is shown that for any meromorphic function $f$ the Julia set $J(f)$ has constant local upper and lower box dimensions, $\bar{d}(J(f))$ and $d(J(f))$ respectively, near all points of $J(f)$ with at most two exceptions. Further, the packing dimension of the Julia set is equal to $\bar{d}(J(f))$. Using this result it is shown that, for any transcendental entire function $f$ in the class $B$ (that is, the class of functions such that the singularities of the inverse function are bounded), both the local upper box dimension and packing dimension of $J(f)$ are equal to 2 . The approach is to show that the subset of the Julia set containing those points that escape to infinity as quickly as possible has local upper box dimension equal to 2 .
\end{abstract}

\section{Introduction}

Let $f$ be a meromorphic function which is not rational of degree one and denote by $f^{n}, n \in \mathbb{N}$, the $n$th iterate of $f$. The set of normality, $F(f)$, is defined to be the set of points, $z \in \mathbb{C}$, such that $\left(f^{n}\right)_{n \in \mathbb{N}}$ is well-defined, meromorphic and forms a normal family in some neighbourhood of $z$. The complement, $J(f)$, of $F(f)$ is called the Julia set of $f$. An introduction to the properties of these sets can be found in, for example, $[\mathbf{1}]$ for rational functions and [2] for transcendental meromorphic functions.

We denote the Hausdorff dimension of a set $A$ by $\operatorname{dim}_{\mathrm{H}} A$, the packing $\operatorname{dimension}$ of $A$ by $\operatorname{dim}_{\mathrm{P}} A$ and, if it exists, the box dimension of $A$ by $\operatorname{dim}_{\mathrm{B}} A$. The upper box dimension $\overline{\operatorname{dim}}_{\mathrm{B}} A$ and the lower box dimension $\underline{\operatorname{dim}}_{\mathrm{B}} A$ are defined for all bounded sets $A$ : if they agree then their common value is the box dimension. These dimensions are related by the inequalities

$$
\operatorname{dim}_{\mathrm{H}} A \leqslant \operatorname{dim}_{\mathrm{P}} A \leqslant \overline{\operatorname{dim}}_{\mathrm{B}} A .
$$

See, for example, [7] for a discussion of these dimensions.

We use the following notation concerning singularities:

$$
S(f)=\left\{z: z \text { is a finite singularity of } f^{-1}\right\},
$$

and then put

$$
B=\{\text { transcendental entire } f: S(f) \text { is bounded }\} .
$$

In [15] we showed that there is a family of transcendental entire functions $f_{K}, K \in$ $\mathbb{N}$, such that the box and packing dimensions of $J\left(f_{K}\right)$ are equal to 2 even though, as $K \rightarrow \infty$, the Hausdorff dimension of $J\left(f_{K}\right)$ tends to 1 , the lowest possible value for the Hausdorff dimension of the Julia set of a transcendental entire function.

Received 30 January 2004; revised 1 September 2004.

2000 Mathematics Subject Classification 30D05, 37F10, 37F15, 37F35, 37F50 (primary). 
This result raises the question as to what possible values can be attained by the box and packing dimensions of the Julia set of a transcendental entire function. In this paper we show that, if $f \in B$, then both the upper box and packing dimensions of the Julia set must be equal to 2 . To do this, we make use of the set

$$
A(f)=\left\{z \text { : there exists } L \in \mathbb{N} \text { such that }\left|f^{n}(z)\right|>M\left(R, f^{n-L}\right) \text {, for } n>L\right\},
$$

which consists of those points that escape to infinity as quickly as possible. Here $M(R, f)=\max _{|z|=R}|f(z)|$ and $R$ can be taken to be any value such that $R>\min _{z \in J(f)}|z|$. The non-empty set $A(f)$ was introduced by Bergweiler and Hinkkanen in $[\mathbf{3}]$ and studied further in $[\mathbf{1 3}]$. We note that, for a transcendental entire function $f$,

each $z \in A(f)$ lies in an unbounded closed connected subset of $A(f)$,

and

$$
A(f) \text { is completely invariant; }
$$

see [13, Theorem 1 and Lemma 2.1]. Eremenko and Lyubich [6] showed that if $f \in B$, then there are no components of $F(f)$ in which the iterates of $f$ tend to infinity. Thus

$$
\text { if } f \in B \text {, then } A(f) \subset J(f) \text {. }
$$

Our main result in this paper is the following.

THEOREM 1.1. If $f \in B$, then

$$
\overline{\operatorname{dim}}_{\mathrm{B}} A(f)=2
$$

and

$$
\operatorname{dim}_{\mathrm{P}} J(f)=\overline{\operatorname{dim}}_{\mathrm{B}} J(f)=2 .
$$

We note that, if $f \in B$, then $\operatorname{dim}_{\mathrm{H}} J(f)>1$; see [14]. There do, however, exist functions in $B$ for which the Hausdorff dimension of the Julia set is arbitrarily close to 1 , for example, the functions $f_{K}$ mentioned earlier.

For a transcendental meromorphic function $f$, both $J(f)$ and $A(f)$ are unbounded and so, strictly speaking, the box dimensions of these sets are not well-defined. In Section 2, however, we prove the following result.

THEOREM 1.2. Let $f$ be a meromorphic function and let $J^{\prime}(f) \subset J(f)$ be such that $f^{-1}\left(J^{\prime}(f)\right) \subset J^{\prime}(f)$. Then there exist non-negative numbers $\bar{d}\left(J^{\prime}(f)\right)$ and $\underline{d}\left(J^{\prime}(f)\right)$ such that, for any bounded open connected set $U$ satisfying $U \cap J^{\prime}(f) \neq \varnothing$ and $\bar{U} \cap E(f)=\varnothing$, we have

$$
\overline{\operatorname{dim}}_{\mathrm{B}}\left(U \cap J^{\prime}(f)\right)=\bar{d}\left(J^{\prime}(f)\right)
$$

and

$$
\underline{\operatorname{dim}}_{\mathrm{B}}\left(U \cap J^{\prime}(f)\right)=\underline{d}\left(J^{\prime}(f)\right) .
$$

Further,

$$
\operatorname{dim}_{P} J(f)=\bar{d}(J(f)) .
$$


In Theorem 1.2,

$$
E(f)=\left\{z: O^{-}(z) \text { is finite }\right\}
$$

where

$$
O^{-}(z)=\left\{w: f^{n}(w)=z, \text { for some } n \in \mathbb{N}\right\} .
$$

The exceptional set $E(f)$ contains at most two points. In many cases, $E(f)$ is contained in the Fatou set $F(f)$, and so Theorem 1.2 gives well-defined local box dimensions on the whole of $J^{\prime}(f)$. In such cases it seems reasonable to define the upper and lower box dimensions of $J^{\prime}(f)$ to be equal to the values $\bar{d}\left(J^{\prime}(f)\right)$ and $\underline{d}\left(J^{\prime}(f)\right)$, respectively. In particular, for this class of functions, the upper box dimension and the packing dimension of the Julia set are equal - this result was proved for rational functions in [18, Theorem 7.1].

In the case where $\bar{d}\left(J^{\prime}(f)\right)=2$, it seems reasonable to define the upper box dimension of $J^{\prime}(f)$ to be equal to 2 since any sensible value for this dimension must lie between $\bar{d}\left(J^{\prime}(f)\right)$ and 2 . This gives another class of functions for which the upper box dimension and the packing dimension of the Julia set are equal.

To prove Theorem 1.1, we take $J^{\prime}(f)=A(f)$ in Theorem 1.2. This is possible by (1.2) and (1.3). We show that, for all functions in the class $B$, the constant $\bar{d}(A(f))$ is equal to 2 . This proves the first part of Theorem 1.1 and it follows that $\bar{d}(J(f))=2$. The second part of Theorem 1.1 then follows from the second part of Theorem 1.2. The procedure by which we obtain the value 2 for $\bar{d}(A(f))$ has two separate stages. The first stage is carried out in Section 4 where we prove the following result.

Proposition 1.3. Let $f \in B$. There exists $R_{0}(f)>0$ such that, if $f^{n}(z) \rightarrow \infty$ as $n \rightarrow \infty$ and $\left|f^{n}(z)\right| \geqslant R_{0}(f)$, for $n=0,1,2, \ldots$, then there exists a bounded neighbourhood $U$ of $z$ with $\bar{U} \cap E(f)=\varnothing$ and

$$
\overline{\operatorname{dim}}_{\mathrm{B}}(U \cap A(f)) \geqslant 2 \varlimsup_{n \rightarrow \infty} \frac{\ln \ln \left|f^{n}(z)\right|}{\ln \left|\left(f^{n}\right)^{\prime}(z) / f^{n}(z)\right|} .
$$

Then, in Section 5, we use Wiman-Valiron theory to prove the following result.

Proposition 1.4. Let $f \in B$. For each $\varepsilon>0$, there exists $z \in A(f)$ with $\left|f^{n}(z)\right| \geqslant R_{0}(f)$, for $n=0,1,2, \ldots$, such that

$$
\varliminf_{n \rightarrow \infty} \frac{\ln \ln \left|f^{n}(z)\right|}{\ln \left|\left(f^{n}\right)^{\prime}(z) / f^{n}(z)\right|} \geqslant 1-\varepsilon .
$$

It follows from these two propositions that, for functions in $B$, the constant $\bar{d}(A(f))$ in Theorem 1.2 must be greater than or equal to $2-2 \varepsilon$, for each $\varepsilon>0$. Thus $\bar{d}(A(f))=2$. Theorem 1.1 then follows from Theorem 1.2.

\section{Proof of Theorem 1.2}

Let $f$ be a meromorphic function and let $J^{\prime}(f)$ be a subset of $J(f)$ such that $f^{-1}\left(J^{\prime}(f)\right) \subset J^{\prime}(f)$. We begin this section by showing that there is a constant local upper box dimension on $J^{\prime}(f) \backslash E(f)$. (The exceptional set $E(f)$ was defined just 
after the statement of Theorem 1.2.) A similar proof can be used to show that there is a constant local lower box dimension on $J^{\prime}(f) \backslash E(f)$.

We use the following well-known property of the Julia set which relates to $E(f)$; see, for example, [2, Section 2]. This is often known as the 'blowing-up property' of the Julia set.

LEMMA 2.1. Let $K$ be a compact set with $K \cap E(f)=\varnothing$ and let $U$ be an open neighbourhood of a point $z \in J(f)$. Then there exists $N \in \mathbb{N}$ such that, for all $n \geqslant N$, we have

$$
f^{n}(U) \supset K
$$

We also use the following basic properties of the upper box dimension. These properties and many others are discussed in detail in, for example, [7].

Lemma 2.2. Let $A$ and $B$ be bounded sets such that $A \subset B$. Then

$$
\overline{\operatorname{dim}}_{\mathrm{B}} A \leqslant \overline{\operatorname{dim}}_{\mathrm{B}} B .
$$

Lemma 2.3. Let $f$ be a Lipschitz map on a bounded set F. Then

$$
\overline{\operatorname{dim}}_{\mathrm{B}} f(F) \leqslant \overline{\operatorname{dim}}_{\mathrm{B}} F .
$$

Using these results, we are able to prove the first part of Theorem 1.2 by showing that $J^{\prime}(f) \backslash E(f)$ has constant local upper box dimension.

LEMMA 2.4. There exists a non-negative number $\bar{d}\left(J^{\prime}(f)\right)$ such that, for any bounded open connected set $U$ satisfying $U \cap J^{\prime}(f) \neq \varnothing$ and $\bar{U} \cap E(f)=\varnothing$,

$$
\overline{\operatorname{dim}}_{\mathrm{B}}\left(U \cap J^{\prime}(f)\right)=\bar{d}\left(J^{\prime}(f)\right) .
$$

Proof. Let $U_{1}$ and $U_{2}$ be bounded open connected sets such that $U_{i} \cap J^{\prime}(f) \neq \varnothing$ and $\bar{U}_{i} \cap E(f)=\varnothing$, for $i=1,2$. It follows from Lemma 2.1 that there exists $N \in \mathbb{N}$ such that $f^{N}\left(U_{1}\right) \supset \bar{U}_{2}$.

Since $\bar{U}_{2}$ is bounded, there exists a set $U_{1}^{\prime} \subset U_{1}$ such that $f^{N}$ is a Lipschitz map from $U_{1}^{\prime}$ onto $U_{2}$. Because $f^{-1}\left(J^{\prime}(f)\right) \subset J^{\prime}(f)$, we have

$$
U_{2} \cap J^{\prime}(f) \subset f^{N}\left(U_{1}^{\prime} \cap J^{\prime}(f)\right),
$$

and so it follows from Lemma 2.2 and Lemma 2.3 that

$$
\overline{\operatorname{dim}}_{\mathrm{B}}\left(U_{2} \cap J^{\prime}(f)\right) \leqslant \overline{\operatorname{dim}}_{\mathrm{B}}\left(U_{1}^{\prime} \cap J^{\prime}(f)\right) \leqslant \overline{\operatorname{dim}}_{\mathrm{B}}\left(U_{1} \cap J^{\prime}(f)\right) .
$$

Since we can interchange the roles of $U_{1}$ and $U_{2}$ in the above argument, we have

$$
\overline{\operatorname{dim}}_{\mathrm{B}}\left(U_{1} \cap J^{\prime}(f)\right)=\overline{\operatorname{dim}}_{\mathrm{B}}\left(U_{2} \cap J^{\prime}(f)\right) .
$$

This completes the proof of Lemma 2.4.

Note that if $U$ is any open set that meets $J^{\prime}(f)$, then $U$ must contain a set $V$ that satisfies all the conditions of Lemma 2.4 and so, by Lemma 2.2, we have the following corollary to Lemma 2.4 . 
Corollary 2.5. Let $U$ be an open set with $U \cap J^{\prime}(f)$ bounded and non-empty. Then

$$
\overline{\operatorname{dim}}_{\mathrm{B}}\left(U \cap J^{\prime}(f)\right) \geqslant \bar{d}\left(J^{\prime}(f)\right) .
$$

In the rest of this section we show that the packing dimension of the Julia set is always equal to $\bar{d}(J(f))$, the local upper box dimension of the Julia set. To do this, we introduce the modified upper box dimension, which we define as

$$
\overline{\operatorname{dim}}_{\mathrm{MB}} A=\inf \left\{\sup _{i \in \mathbb{N}} \overline{\operatorname{dim}}_{\mathrm{B}} A_{i}: A \subset \bigcup_{i=1}^{\infty} A_{i}\right\},
$$

where the infimum is over all possible countable covers of $A$ with bounded sets $A_{i}$, $i \in \mathbb{N}$. By modifying the method described by Falconer [7, Sections 3.3 and 3.4], we shall show that the modified upper box dimension of the Julia set is always equal to $\bar{d}(J(f))$. The modified upper box dimension is actually equal to the packing dimension (see [7, Proposition 3.8]) and so this is sufficient to complete the proof of Theorem 1.2. (Note that, in [7, Proposition 3.8], the set $A$ is assumed to be bounded but the proof applies equally well if the set $A$ is unbounded.)

Our proof that $\overline{\operatorname{dim}}_{\mathrm{MB}} J(f)=\bar{d}(J(f))$ has two steps. We begin by proving the following result.

LEMMA 2.6.

$$
\overline{\operatorname{dim}}_{\mathrm{MB}} J(f) \leqslant \bar{d}(J(f))
$$

Proof. For each $n \in \mathbb{N}$, put

$$
U_{n}=\{z:|z|<n,|z-w|>1 / n \text { for each } w \in E(f)\} .
$$

(Recall that $E(f)$ contains at most two points.) Then each $U_{n}$ is a bounded open connected set, $\bar{U}_{n} \cap E(f)=\varnothing$ and, for sufficiently large $n \in \mathbb{N}$, we have $U_{n} \cap J(f) \neq$ $\varnothing$. It follows from Lemma 2.4 that

$$
\overline{\operatorname{dim}}_{\mathrm{B}}\left(U_{n} \cap J(f)\right)=\bar{d}(J(f)),
$$

for large values of $n \in \mathbb{N}$, and so

$$
\overline{\operatorname{dim}}_{\mathrm{B}}\left(U_{n} \cap J(f)\right) \leqslant \bar{d}(J(f)),
$$

for every $n \in \mathbb{N}$.

Since

$$
J(f)=\bigcup_{n=1}^{\infty}\left(U_{n} \cap J(f)\right) \cup(E(f) \cap J(f))
$$

and $E(f)$ contains at most two points, it follows from (2.1) and (2.2) that

$$
\overline{\operatorname{dim}}_{\mathrm{MB}} J(f) \leqslant \sup _{n \in \mathbb{N}} \overline{\operatorname{dim}}_{\mathrm{B}}\left(U_{n} \cap J(f)\right)=\bar{d}(J(f)) .
$$

This completes the proof of Lemma 2.6.

We now show that the opposite inequality is true, thus obtaining the equality that we need. 
LEMMA 2.7.

$$
\overline{\operatorname{dim}}_{\mathrm{MB}} J(f) \geqslant \bar{d}(J(f))
$$

Proof. In order to obtain an estimate for the modified upper box dimension of $J(f)$, it is sufficient to consider countable covers of $J(f)$ with compact sets since the upper box dimension of a set is equal to the upper box dimension of its closure; see, [7, Proposition 3.4]. Suppose that $J(f) \subset \bigcup_{i=1}^{\infty} A_{i}$, where $A_{i}$ is a compact set for each $i \in \mathbb{N}$. Since $J(f)$ is complete, it follows from Baire's category theorem that there exists $j \in \mathbb{N}$ and an open set $U$ such that $U \cap J(f) \neq \varnothing$ and

$$
U \cap J(f) \subset A_{j} .
$$

Thus, by Lemma 2.2 and Corollary 2.5,

$$
\overline{\operatorname{dim}}_{\mathrm{B}} A_{j} \geqslant \overline{\operatorname{dim}}_{\mathrm{B}}(U \cap J(f)) \geqslant \bar{d}(J(f)),
$$

so

$$
\sup _{i \in \mathbb{N}} \overline{\operatorname{dim}}_{\mathrm{B}} A_{i} \geqslant \bar{d}(J(f))
$$

Since this is true for every countable cover with compact sets (and hence for every countable cover with bounded sets), the result now follows from the definition of the modified upper box dimension.

Note that the methods of proof in this section can also be used to show that the Hausdorff dimension of the Julia set of a meromorphic function is locally constant. A similar result (and proof) holds for the packing dimension.

THEOREM 2.8. Let $f$ be a meromorphic function and let $U$ be an open set that meets $J(f)$. Then

$$
\operatorname{dim}_{\mathrm{H}}(U \cap J(f))=\operatorname{dim}_{\mathrm{H}} J(f) .
$$

Proof. First note that the proof of Lemma 2.4 can be used to give a similar result about Hausdorff dimension. Therefore, since Hausdorff dimension is stable under countable unions and $E(f)$ contains at most two points, it follows from $(2.3)$ that (2.4) holds for any bounded open connected set $U$ satisfying $U \cap J(f) \neq \varnothing$ and $\bar{U} \cap E(f)=\varnothing$. The result follows since any open set $U$ that meets $J(f)$ contains a bounded open connected set $V$ such that $V \cap J(f) \neq \varnothing$ and $\bar{V} \cap E(f)=\varnothing$, and

$$
\operatorname{dim}_{\mathrm{H}} J(f)=\operatorname{dim}_{\mathrm{H}}(V \cap J(f)) \leqslant \operatorname{dim}_{\mathrm{H}}(U \cap J(f)) \leqslant \operatorname{dim}_{\mathrm{H}} J(f) .
$$

\section{Properties of functions in the class $B$}

In this section we give some results concerning the class $B$, which will be useful in the proof of Theorem 1.1.

The first result was proved by Eremenko and Lyubich [6, Lemma 1]. Here, and later, $B(z, r)$ denotes the open disc of radius $r$, centred at $z$.

LEMma 3.1. Let $f \in B$ with $S(f) \subset B(0, R / 2)$ and let $U$ be a component of $f^{-1}(\{w:|w|>R\})$. Then $U$ is simply connected and bounded by a single unbounded simple curve. 
Further, if $0 \notin U$ and $V=\ln (U)$, for some branch of $\ln$ analytic in $U$, then there exists a map $F$ such that, for all $t \in V, f\left(e^{t}\right)=e^{F(t)}$ and

$$
\left|F^{\prime}(t)\right| \geqslant \frac{\Re(F(t))-\ln R}{4 \pi} .
$$

The next result is a simple corollary of Lemma 3.1 .

Corollary 3.2. Let $f \in B$. There exists $R_{1}(f)>0$ such that, if $|f(z)|>R_{1}(f)$, then

$$
\left|f^{\prime}(z)\right|>\frac{|f(z)| \ln |f(z)|}{8 \pi|z|} .
$$

By repeatedly applying Corollary 3.2, we obtain the following.

Corollary 3.3. Let $f \in B$. If $\left|f^{k}(z)\right|>\max \left\{R_{1}(f), e^{8 \pi}\right\}$, for $0 \leqslant k \leqslant n$, then

$$
\left|\left(f^{n}\right)^{\prime}(z)\right|>\frac{\left|f^{n}(z)\right| \ln \left|f^{n}(z)\right|}{8 \pi|z|} .
$$

The next result in this section was proved in [14, Lemma 2.6].

LEMmA 3.4. Let $f \in B$. There exists $R_{2}(f) \geqslant R_{1}(f)$ such that, if $\left|f^{k}(z)\right| \geqslant$ $R_{2}(f)$, for $0 \leqslant k \leqslant n$, then the branch $g$ of $f^{-n}$ that maps $f^{n}(z)$ to $z$ is univalent in $B\left(f^{n}(z),\left|f^{n}(z)\right| / 4\right)$.

We also require the following estimate for the growth of the maximum modulus of a function in the class $B$. In view of the fact that $f$ is bounded on a curve tending to infinity (see the first part of Lemma 3.1), this estimate can be deduced from the $\cos \pi \rho$ theorem in the case $\rho=\frac{1}{2}$ (see [10, p. 302]), but we include a proof here for the sake of completeness. For simplicity, we use $M(r)$ to denote $M(r, f)=\max _{|z|=r}|f(z)|$.

LEMma 3.5. If $f \in B$, then there exist constants $c=c(f)>0$ and $r_{0}=r_{0}(f)>1$ such that

$$
\ln M(r) \geqslant c r^{1 / 2} \geqslant(\ln 2 r)^{2}, \quad r \geqslant r_{0}
$$

Proof. Let $R>1$ have the property that $S(f) \subset B(0, R / 2)$, and let $U$ be a component of $f^{-1}(\{w:|w|>R\})$. It follows from the first part of Lemma 3.1 that $U$ is simply connected and bounded by a single unbounded simple curve. By applying a linear change of variables and the maximum principle, we may assume that $0 \in U$.

Let $U_{r}$ be the component of $U \cap B(0, r)$ containing 0 , and $\omega_{r}$ be the harmonic measure of $\partial U_{r} \cap\{z:|z|=r\}$ with respect to $U_{r}$. Then let $r \theta(r)$ be the linear measure of $\partial U_{r} \cap\{z:|z|=r\}$. There exists $r_{0}=r_{0}(f)>0$ such that $\theta(r)=2 \pi$ if and only if $r \leqslant r_{0} / 2$. We put

$$
\theta^{*}(r)= \begin{cases}\theta(r) & r>r_{0} / 2 \\ \infty & r \leqslant r_{0} / 2\end{cases}
$$


Then, by $[\mathbf{1 7}$, p. 112], we have

$$
\omega_{r}(0) \leqslant 3 \sqrt{2} \exp \left(-\pi \int_{0}^{r / 2} \frac{d t}{t \theta^{*}(t)}\right), \quad r>0 .
$$

Now put $u(z)=\ln ^{+}(|f(z)| / R)$. Then $u$ is a non-negative subharmonic function equal to 0 on $\partial U_{r} \cap B(0, r)$, since $|f(z)|=R$ here, and bounded above by $\ln ^{+}(M(r) / R)$ on $\partial U_{r} \cap\{z:|z|=r\}$. Also $u(0)>0$, since $0 \in U$. By the maximum principle for subharmonic functions, $u(0) \leqslant \omega_{r}(0) \ln ^{+}(M(r) / R)$. Thus, if $r>r_{0}$, then

$$
\ln (M(r) / R) \geqslant \frac{u(0)}{3 \sqrt{2}} \exp \left(\pi \int_{0}^{r / 2} \frac{d t}{t \theta^{*}(t)}\right)=\frac{u(0)}{3 \sqrt{2}} \exp \left(\pi \int_{r_{0} / 2}^{r / 2} \frac{d t}{t \theta(t)}\right) .
$$

Now $\theta(t) \leqslant 2 \pi$, for all $t>0$, and so

$$
\pi \int_{r_{0} / 2}^{r / 2} \frac{d t}{t \theta(t)} \geqslant \frac{1}{2} \ln \left(r / r_{0}\right)
$$

Thus, for $r \geqslant r_{0}$,

$$
\ln (M(r) / R) \geqslant \frac{u(0)}{3 \sqrt{2}} \exp \left(\frac{1}{2} \ln \left(r / r_{0}\right)\right)
$$

and so

$$
\ln M(r) \geqslant c r^{1 / 2}, \quad r \geqslant r_{0},
$$

as required. The second inequality in Lemma 3.5 follows immediately.

\section{Proof of Proposition 1.3}

We begin this section with a formal definition of the box dimension of a bounded set $A \subset \mathbb{C}$. Suppose that we have a grid composed of lines parallel to the axes such that each box in the grid has sides of length $d$. We count the number $N_{d}(A)$ of boxes in this grid that meet the set $A$. The box dimension of $A$ is defined to be

$$
\operatorname{dim}_{\mathrm{B}} A=\lim _{d \rightarrow 0} \frac{\ln N_{d}(A)}{-\ln d},
$$

if this limit exists. There are several equivalent definitions of the box dimension of a set; for more details see, for example, [7]. The upper and lower box dimensions are obtained by replacing $\lim _{d \rightarrow 0}$ in this definition with $\varlimsup_{d \rightarrow 0}$ and $\underline{\lim }_{d \rightarrow 0}$ respectively.

To prove Proposition 1.3, we use some of the properties of the set $A(f)$. In particular, it follows from $(1.1)$ that $A(f)$ contains an unbounded closed connected set, $\Gamma$ say.

Now let $f \in B$ and take $R>0$ with

$$
S(f) \cup E(f) \subset B(0, R / 2) \text { and } \Gamma \cap B(0, R / 2) \neq \varnothing .
$$

Also, choose positive constants $c_{0}, c_{1}$ and $C_{0}$ such that

$$
c_{0} \geqslant 4 \pi L, \quad c_{1} \leqslant \frac{1}{96 \pi L} \quad \text { and } \quad C_{0} \leqslant \frac{c_{1}}{2 L},
$$

where $L=81$. Then let $z_{0}$ be such that $f^{n}\left(z_{0}\right) \rightarrow \infty$ as $n \rightarrow \infty$ and

$$
\left|f^{n}\left(z_{0}\right)\right| \geqslant R_{0}(f)=\max \left\{R_{2}(f), \exp \left(c_{0} / C_{0}\right),(R / 2)^{4}\right\}, \quad \text { for } n=0,1,2, \ldots,
$$

where $R_{2}(f)$ is as defined in Lemma 3.4. 


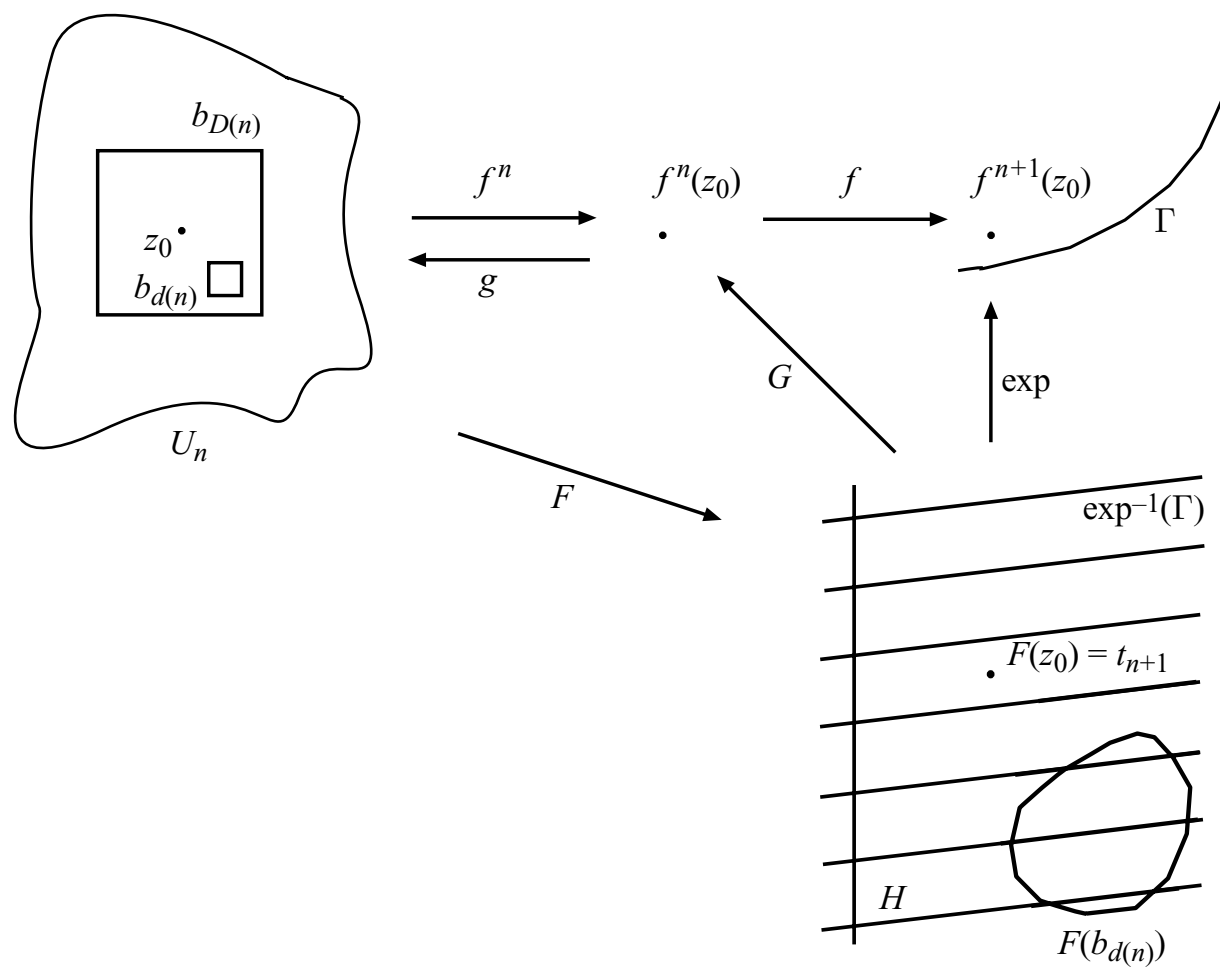

Figure 1.

We need to show that there exists a bounded neighbourhood $U$ of $z_{0}$ with $\bar{U} \cap E(f)=\varnothing$ and

$$
\overline{\operatorname{dim}}_{\mathrm{B}}(U \cap A(f)) \geqslant 2 \varlimsup_{n \rightarrow \infty} \frac{\ln \ln \left|f^{n}\left(z_{0}\right)\right|}{\ln \left|\left(f^{n}\right)^{\prime}\left(z_{0}\right) / f^{n}\left(z_{0}\right)\right|} .
$$

To do this, we let $b_{D(n)}$ denote the box centred at $z_{0}$ with sides parallel to the axes and of length

$$
D(n)=C_{0} \frac{\left|f^{n+1}\left(z_{0}\right)\right| \ln \left|f^{n+1}\left(z_{0}\right)\right|}{\left|\left(f^{n+1}\right)^{\prime}\left(z_{0}\right)\right|} .
$$

We then let $b_{d(n)}$ denote a box within $b_{D(n)}$ centred at a point, $z_{d(n)}$ say, with sides parallel to the axes and of length

$$
d(n)=\frac{c_{0}\left|f^{n+1}\left(z_{0}\right)\right|}{\left|\left(f^{n+1}\right)^{\prime}\left(z_{0}\right)\right|} .
$$

The fact that $d(n) \leqslant D(n)$, for $n=1,2, \ldots$, follows from (4.3).

The key step in the proof of Proposition 1.3 is the following result.

Lemma 4.1. For $n=1,2, \ldots$, any box of the form $b_{d(n)}$ meets $A(f)$.

Proof. Figure 1 illustrates how we prove Lemma 4.1.

For $n=1,2, \ldots$, we shall find a neighbourhood $U_{n}$ of $z_{0}$ and a univalent branch $F$ of $\ln f^{n+1}$, defined on $U_{n}$, such that $b_{D(n)} \subset U_{n}$,

$$
F\left(b_{D(n)}\right) \subset B\left(F\left(z_{0}\right), c_{1}\left|F\left(z_{0}\right)\right|\right) \subset H
$$


where $c_{1}$ satisfies (4.2) and $H=\{t: \Re(t)>\ln (R / 2)\}$, and

$$
F\left(b_{d(n)}\right) \supset B\left(F\left(z_{d(n)}\right), 2 \pi\right) .
$$

It follows from (4.6), (4.7) and (4.1) that $F\left(b_{d(n)}\right)$ meets $\exp ^{-1}(\Gamma)$ and so $f^{n+1}\left(b_{d(n)}\right)$ meets $\Gamma$, for each $n \in \mathbb{N}$. Lemma 4.1 then follows by the complete invariance of $A(f)$; see (1.2).

We now prove (4.6) and (4.7). In doing so, we make much use of the following result known as Koebe's distortion theorem; see, for example [4, Theorem 2.5].

Lemma 4.2. If $f$ is univalent in $B(z, r)$, then, for $0 \leqslant s<r$,

$$
\sup _{v, w \in B(z, s)}\left|\frac{f^{\prime}(v)}{f^{\prime}(w)}\right| \leqslant\left(\frac{r+s}{r-s}\right)^{4}=L(s / r) .
$$

Note that $L(1 / 2)=81=L$. In particular, under the hypotheses of Lemma 4.2 , we have

$$
B\left(f(z),\left|f^{\prime}(z)\right| r /(2 L)\right) \subset f(B(z, r / 2)) \subset B\left(f(z), L\left|f^{\prime}(z)\right| r / 2\right) .
$$

Now choose $t_{n+1}$ so that $f^{n+1}\left(z_{0}\right)=e^{t_{n+1}}$ and $-\pi<\Im\left(t_{n+1}\right) \leqslant \pi$. Note that

$$
\ln \left|f^{n+1}\left(z_{0}\right)\right| \leqslant\left|t_{n+1}\right|<(3 / 2) \ln \left|f^{n+1}\left(z_{0}\right)\right|,
$$

by (4.3). Let $G$ denote the branch of $f^{-1}(\exp )$ that maps $t_{n+1}$ to $f^{n}\left(z_{0}\right)$ and let $g$ denote the branch of $f^{-n}$ that maps $f^{n}\left(z_{0}\right)$ to $z_{0}$. Since $S(f) \subset B(0, R / 2)$, the branch $G$ can be analytically continued along all the paths from $t_{n+1}$ in $H=$ $\{t: \Re(t)>\ln (R / 2)\}$ to give a single-valued analytic function in $H$, by the monodromy theorem. Two cases can then arise (see [11, p. 283] or [12, Section 2]) as follows.

(a) The function $G$ is univalent in $H$.

(b) The function $G$ is periodic in $H$, with period $2 \pi i m$ for some minimum positive integer $m$.

In the present situation case (b) cannot occur. Indeed, case (b) implies that $G(t)=\phi\left(e^{t / m}\right)$, where $\phi$ is univalent in $\left\{s:|s|>(R / 2)^{1 / m}\right\}$ with expansion there of the form

$$
\phi(s)=a_{1} s+a_{0}+a_{-1} s^{-1}+\ldots
$$

Thus, if $z=G(t), t \in H$, then we have $z \in \phi\left(\left\{s:|s|>(R / 2)^{1 / m}\right\}\right)$ and

$$
f(z)=e^{t}=\left(\phi^{-1}(z)\right)^{m} .
$$

If $a_{1} \neq 0$, then $f(z) \sim a_{1}^{-m} z^{m}$ as $z \rightarrow \infty$, which is impossible since $f$ has an essential singularity at $\infty$. Hence $a_{1}=0$ and so $\phi(\infty)=a_{0}$. Thus $a_{0}$ is a pole of $f$, which is impossible. We deduce that $G$ is univalent in $H$.

It follows from (4.3) and (4.9) that $B\left(t_{n+1},\left|t_{n+1}\right| / 2\right) \subset H$, so $G$ is univalent in $B\left(t_{n+1},\left|t_{n+1}\right| / 2\right)$. Since

$$
G^{\prime}(t)=\frac{f(G(t))}{f^{\prime}(G(t))}
$$


and $G\left(t_{n+1}\right)=f^{n}\left(z_{0}\right)$, we deduce from (4.8), (4.9), Corollary 3.2 and (4.2) that

$$
\begin{aligned}
G\left(B\left(t_{n+1}, 2 c_{1}\left|t_{n+1}\right|\right)\right) & \subset B\left(f^{n}\left(z_{0}\right), 2 c_{1} L\left|t_{n+1}\right|\left|\frac{f^{n+1}\left(z_{0}\right)}{f^{\prime}\left(f^{n}\left(z_{0}\right)\right)}\right|\right) \\
& \subset B\left(f^{n}\left(z_{0}\right), 3 c_{1} L \ln \left|f^{n+1}\left(z_{0}\right)\right|\left|\frac{f^{n+1}\left(z_{0}\right)}{f^{\prime}\left(f^{n}\left(z_{0}\right)\right)}\right|\right) \\
& \subset B\left(f^{n}\left(z_{0}\right),\left|f^{n}\left(z_{0}\right)\right| / 4\right) .
\end{aligned}
$$

In view of (4.3), it follows from Lemma 3.4 that $g$ is univalent in $B\left(f^{n}\left(z_{0}\right)\right.$, $\left.\left|f^{n}\left(z_{0}\right)\right| / 4\right)$ and so $g \circ G$ is univalent in $B\left(t_{n+1}, 2 c_{1}\left|t_{n+1}\right|\right)$. Since

$$
(g \circ G)^{\prime}(t)=\frac{f^{n+1}((g \circ G)(t))}{\left(f^{n+1}\right)^{\prime}((g \circ G)(t))},
$$

and $g\left(G\left(t_{n+1}\right)\right)=z_{0}$, we deduce from (4.8), (4.9), (4.4) and (4.2) that

$$
\begin{aligned}
(g \circ G)\left(B\left(t_{n+1}, c_{1}\left|t_{n+1}\right|\right)\right) & \supset B\left(z_{0}, c_{1}\left|t_{n+1}\right|\left|\frac{f^{n+1}\left(z_{0}\right)}{L\left(f^{n+1}\right)^{\prime}\left(z_{0}\right)}\right|\right) \\
& \supset B\left(z_{0}, c_{1} \ln \left|f^{n+1}\left(z_{0}\right)\right|\left|\frac{f^{n+1}\left(z_{0}\right)}{L\left(f^{n+1}\right)^{\prime}\left(z_{0}\right)}\right|\right) \\
& \supset B\left(z_{0}, 2 D(n)\right) \supset b_{D(n)} .
\end{aligned}
$$

This shows that (4.6) is true with $U_{n}=(g \circ G)\left(B\left(t_{n+1}, 2 c_{1}\left|t_{n+1}\right|\right)\right)$, where $F$ is the inverse function of $g \circ G$ defined on $U_{n}$. In particular, note that $F\left(z_{0}\right)=t_{n+1}$.

Since $F$ is univalent in $B\left(z_{0}, 2 D(n)\right), b_{d(n)} \subset b_{D(n)} \subset B\left(z_{0}, D(n)\right)$ and $F^{\prime}(z)=$ $\left(f^{n+1}\right)^{\prime}(z) / f^{n+1}(z)$, we deduce from $(4.8),(4.5)$ and $(4.2)$ that

$$
\begin{aligned}
F\left(b_{d(n)}\right) & \supset F\left(B\left(z_{d(n)}, d(n) / 2\right)\right) \\
& \supset B\left(F\left(z_{d(n)}\right), d(n)\left|F^{\prime}\left(z_{0}\right)\right| /(2 L)\right) \\
& =B\left(F\left(z_{d(n)}\right), c_{0} /(2 L)\right) \\
& \supset B\left(F\left(z_{d(n)}\right), 2 \pi\right) .
\end{aligned}
$$

Thus (4.7) holds. This completes the proof of Lemma 4.1.

Now let $U=B\left(z_{0}, 16 \pi C_{0}\left|z_{0}\right|\right)$, so $b_{D(n)} \subset B\left(z_{0}, 2 D(n)\right) \subset U$, for $n \in \mathbb{N}$, by Corollary 3.3. It follows from (4.1), (4.2) and (4.3) that $\bar{U} \cap E(f)=\varnothing$. We apply the definition of upper box dimension given at the beginning of this section to the set $A=U \cap A(f)$. By Lemma 4.1, any box of the form $b_{d(n)}$ meets $A$. By estimating the maximum number of such disjoint boxes that can fit inside $b_{D(n)}$, we obtain

$$
N_{d(n)}(A) \geqslant(D(n) / 2 d(n))^{2} \geqslant\left(C_{0} / 2 c_{0}\right)^{2}\left(\ln \left|f^{n+1}\left(z_{0}\right)\right|\right)^{2} .
$$

Since $f^{n}\left(z_{0}\right) \rightarrow \infty$ as $n \rightarrow \infty$, it follows from Corollary 3.3 that $d(n) \rightarrow 0$ as $n \rightarrow \infty$, and so

$$
\begin{aligned}
\overline{\operatorname{dim}}_{\mathrm{B}} A & \geqslant \varlimsup_{d(n) \rightarrow 0} \frac{\ln N_{d(n)}(A)}{-\ln d(n)} \\
& \geqslant \varlimsup_{n \rightarrow \infty} \frac{2 \ln \ln \left|f^{n+1}\left(z_{0}\right)\right|+\ln \left(C_{0} / 2 c_{0}\right)^{2}}{\ln \left|\left(f^{n+1}\right)^{\prime}\left(z_{0}\right) / f^{n+1}\left(z_{0}\right)\right|-\ln c_{0}} \\
& =2 \varlimsup_{n \rightarrow \infty} \frac{\ln \ln \left|f^{n+1}\left(z_{0}\right)\right|}{\ln \left|\left(f^{n+1}\right)^{\prime}\left(z_{0}\right) / f^{n+1}\left(z_{0}\right)\right|} .
\end{aligned}
$$

This completes the proof of Proposition 1.3. 


\section{Proof of Proposition 1.4}

Let $f$ be a function in the class $B$. It follows from Corollary 3.3 that

$$
\varlimsup_{n \rightarrow \infty} \frac{\ln \ln \left|f^{n}(z)\right|}{\ln \left|\left(f^{n}\right)^{\prime}(z) / f^{n}(z)\right|} \leqslant 1,
$$

whenever $f^{n}(z) \rightarrow \infty$ as $n \rightarrow \infty$. In this section we show that there are points $z \in A(f)$ for which it is possible to obtain a lower bound for the expression

$$
\varliminf_{n \rightarrow \infty} \frac{\ln \ln \left|f^{n}(z)\right|}{\ln \left|\left(f^{n}\right)^{\prime}(z) / f^{n}(z)\right|}
$$

which is arbitrarily close to 1 . This is sufficient to prove Proposition 1.4.

Our proof is based on a method introduced by Eremenko [5] to construct a point $z$ such that $f^{n}(z) \rightarrow \infty$ as $n \rightarrow \infty$, where $f$ is a transcendental entire function. If $f(z)=\sum_{n=0}^{\infty} a_{n} z^{n}$, then we define

$$
\mu(r)=\sup _{n}\left|a_{n}\right| r^{n}=\left|a_{N}\right| r^{N}, \quad r>0,
$$

to be the maximal term and call $N=N(r)$ the central index; if (5.1) holds for several $N$, then we take $N(r)$ to be the largest of these. Note that $N(r)$ is increasing and $N(r) \rightarrow \infty$ as $r \rightarrow \infty$. Wiman-Valiron theory uses $\mu(r)$ to give results about the behaviour of $f$ near those points $w(r), r>0$, that satisfy

$$
|f(w(r))|=M(r, f) \text { and }|w(r)|=r .
$$

For simplicity, we will denote $M(r, f)$ by $M(r)$ for the remainder of this section.

Let $f$ be a transcendental entire function and $\alpha>\frac{1}{2}$. The key result of WimanValiron theory used by Eremenko is that, if

$$
|z-w(r)|<r(N(r))^{-\alpha},
$$

then

$$
f(z)=\left(\frac{z}{w(r)}\right)^{N(r)} f(w(r))\left(1+\varepsilon_{1}\right)
$$

and

$$
f^{\prime}(z)=N(r)\left(\frac{z}{w(r)}\right)^{N(r)} f(w(r))(w(r))^{-1}\left(1+\varepsilon_{2}\right),
$$

where, for $i=1,2$, the error terms $\varepsilon_{i}=\varepsilon_{i}(r, z) \rightarrow 0$ uniformly with respect to $z$ as $r \rightarrow \infty, r \notin E$. The exceptional set $E=E(f, \alpha)$ is of finite logarithmic measure; that is, $\int_{E}(1 / t) d t<\infty$. This result is given in [8, p. 277]; see also [9], [10] and [19] for other accounts of Wiman-Valiron theory.

Eremenko [5, proof of Theorem 1] used (5.4) and (5.5), with a fixed $\alpha \in\left(\frac{1}{2}, 1\right)$, to construct a sequence $r_{n}>2, n=1,2, \ldots$, and an orbit $z_{n}=f^{n-1}\left(z_{1}\right), n=$ $1,2, \ldots$, such that

$$
\begin{gathered}
2 r_{n}<\frac{1}{2} M\left(r_{n}\right)<r_{n+1}<2 M\left(r_{n}\right), \quad n=1,2, \ldots \\
r_{n} \notin E, \quad n=1,2, \ldots
\end{gathered}
$$

and

$$
\max \left\{|\ln | \frac{z_{n}}{w\left(r_{n}\right)}||,\left|\arg \frac{z_{n}}{w\left(r_{n}\right)}\right|\right\}<\frac{5}{N\left(r_{n}\right)}<10^{-3}, \quad n=1,2, \ldots
$$


Note that in Eremenko's construction $r_{n}$ is chosen inductively subject only to (5.6) and (5.7), and the restriction on $r_{1}$ that

$$
\int_{E \cap\left[r_{1}, \infty\right)} \frac{d t}{t}<1
$$

The conditions (5.6) and (5.8) imply that $z_{1} \in A(f)$, using an argument similar to that in $[\mathbf{3}$, p. 570].

In our construction we choose the sequence $r_{n}$ to satisfy all the above conditions, and several others, most of which are associated with the following lemma. This lemma can be found in [9, Theorem 6 and proof of (6.20)]; see also [10, Theorem 6.23 and Lemma 6.15].

LEMMA 5.1. Let $f$ be a transcendental entire function.

(a) For all $r$ apart from a set of finite logarithmic measure, we have

$$
M(r)<\mu(r)^{2} .
$$

(b) Suppose that $\delta>0$. Then, for all $r$ apart from a set of finite logarithmic measure, we have

$$
N(r)<(\ln \mu(r))^{1+\delta} \leqslant(\ln M(r))^{1+\delta} .
$$

The results in parts (a) and (b) of Lemma 5.1 are not best possible, but they are sufficient for our purposes.

Given $\delta, 0<\delta<\frac{1}{2}$, we can choose the sequences $r_{n}$ and $z_{n}$ so that (5.6), (5.7) and (5.8) hold, and in addition so that

$$
M\left(r_{n}\right)<\mu\left(r_{n}\right)^{2}, \quad n=1,2, \ldots,
$$

by Lemma 5.1(a),

$$
N\left(r_{n}\right)<\left(\ln M\left(r_{n}\right)\right)^{1+\delta}, \quad n=1,2, \ldots,
$$

by Lemma 5.1(b),

$$
r_{n}>r_{0}, \quad n=1,2 \ldots,
$$

where $r_{0}$ is the constant in Lemma 3.5,

$$
\ln M\left(r_{n}\right) \geqslant 2^{1 / \delta}, \quad n=1,2, \ldots,
$$

since $M\left(r_{n}\right) \rightarrow \infty$ as $n \rightarrow \infty$, and

$$
\left|\frac{f^{\prime}\left(z_{n}\right)}{f\left(z_{n}\right)}\right|=\frac{N\left(r_{n}\right)}{\left|z_{n}\right|}\left|1+\sigma_{n}\right|, \quad \text { where }\left|\sigma_{n}\right| \leqslant \frac{1}{2}, n=1,2, \ldots,
$$

by (5.3), (5.4), (5.5) and (5.8). Note that the sequence $r_{n}$ can be chosen so that (5.10) and (5.11) hold because both exceptional sets in Lemma 5.1 are of finite logarithmic measure and (5.6) can be satisfied as long as $r_{1}$ is chosen large enough that these two new exceptional sets satisfy a condition of the form of (5.9).

Using these conditions, we establish the following estimate.

LEMma 5.2. If $f \in B, 0<\delta<1 / 2$, and the sequences $r_{n}$ and $z_{n}=f^{n-1}\left(z_{1}\right)$, $n=1,2, \ldots$, are chosen as above, then

$$
\varliminf_{n \rightarrow \infty} \frac{\ln \ln \left|f^{n}\left(z_{1}\right)\right|}{\ln \left|\left(f^{n}\right)^{\prime}\left(z_{1}\right) / f^{n}\left(z_{1}\right)\right|} \geqslant \frac{1}{1+2 \delta} .
$$


Proof. In view of (5.14), we have, for $n=1,2, \ldots$,

$$
\left|\left(f^{n}\right)^{\prime}\left(z_{1}\right)\right|=\left|f^{\prime}\left(f^{n-1}\left(z_{1}\right)\right) \ldots f^{\prime}\left(z_{1}\right)\right|=\frac{\left|f^{n}\left(z_{1}\right)\right|}{\left|z_{1}\right|} \prod_{k=1}^{n}\left|1+\sigma_{k}\right| N\left(r_{k}\right),
$$

So

$$
\frac{\ln \left|\left(f^{n}\right)^{\prime}\left(z_{1}\right) / f^{n}\left(z_{1}\right)\right|}{\ln N\left(r_{n}\right)}=1+\frac{\ln \left(\left(\left|1+\sigma_{n}\right| /\left|z_{1}\right|\right) \prod_{k=1}^{n-1}\left|1+\sigma_{k}\right| N\left(r_{k}\right)\right)}{\ln N\left(r_{n}\right)} .
$$

Now we estimate $N(r)$ using the fact [10, proof of Theorem 6.23] that

$$
\ln \mu(r)=\ln \mu\left(r_{0}\right)+\int_{r_{0}}^{r} \frac{N(t)}{t} d t, \quad r>r_{0} .
$$

Since $N(r)$ is increasing and $r_{0}>1$, we deduce from this equation that

$$
N(r) \geqslant \frac{\ln \mu(r)-\ln \mu\left(r_{0}\right)}{\ln r}, \quad r>r_{0}
$$

In this inequality we take $r=r_{n}$ and use the estimates (5.10) and (5.11) to give

$$
\frac{\ln M\left(r_{n}\right)-a}{2 \ln r_{n}} \leqslant N\left(r_{n}\right) \leqslant\left(\ln M\left(r_{n}\right)\right)^{1+\delta}, \quad n=1,2, \ldots,
$$

where $a=2 \ln \mu\left(r_{0}\right)$. Using the right-hand side of (5.16) together with (5.13) and $(5.14)$, we obtain $1+\sigma_{n} \mid N\left(r_{n}\right) \leqslant 2\left(\ln M\left(r_{n}\right)\right)^{1+\delta} \leqslant\left(\ln M\left(r_{n}\right)\right)^{1+2 \delta}, \quad n=1,2, \ldots$

Thus

$$
\prod_{k=1}^{n-1}\left|1+\sigma_{k}\right| N\left(r_{k}\right) \leqslant\left(\prod_{k=1}^{n-1} \ln M\left(r_{k}\right)\right)^{1+2 \delta}, \quad n=1,2, \ldots
$$

Next, by the second inequality in Lemma 3.5, (5.6) and (5.12), we have

$$
\ln M\left(r_{n-1}\right) \geqslant\left(\ln M\left(r_{n-2}\right)\right)^{2} \geqslant\left(\ln M\left(r_{n-3}\right)\right)^{4} \geqslant \ldots,
$$

So

$$
\prod_{k=1}^{n-1} \ln M\left(r_{k}\right) \leqslant\left(\ln M\left(r_{n-1}\right)\right)^{1+1 / 2+1 / 4+\ldots} \leqslant\left(\ln M\left(r_{n-1}\right)\right)^{2} .
$$

It follows from (5.18), (5.19), (5.6), the left-hand side of (5.16), and Lemma 3.5 that

$$
\begin{aligned}
\frac{\ln \left(\prod_{k=1}^{n-1}\left|1+\sigma_{k}\right| N\left(r_{k}\right)\right)}{\ln N\left(r_{n}\right)} & \leqslant \frac{\ln \left(\ln M\left(r_{n-1}\right)\right)^{2(1+2 \delta)}}{\ln \left(\ln M\left(r_{n}\right)-a\right)-\ln \left(2 \ln r_{n}\right)} \\
& \leqslant \frac{2(1+2 \delta) \ln \ln \left(2 r_{n}\right)}{\ln \left(c r_{n}^{1 / 2}-a\right)-\ln \left(2 \ln r_{n}\right)} \\
& \rightarrow 0 \quad \text { as } n \rightarrow \infty .
\end{aligned}
$$

The left-hand side of the above inequality is positive since $\left|1+\sigma_{n}\right| N\left(r_{n}\right)>1$, for $n=1,2, \ldots$, by (5.8) and (5.14), and so we conclude from (5.15) that

$$
\lim _{n \rightarrow \infty} \frac{\ln \left|\left(f^{n}\right)^{\prime}\left(z_{1}\right) / f^{n}\left(z_{1}\right)\right|}{\ln N\left(r_{n}\right)}=1 .
$$


To complete the proof of Lemma 5.2, we deduce from (5.17) that

$$
\ln \left|1+\sigma_{n}\right|+\ln N\left(r_{n}\right) \leqslant(1+2 \delta) \ln \ln M\left(r_{n}\right), \quad n=1,2, \ldots,
$$

and from (5.6) and (5.8) that $\left|f^{n}\left(z_{1}\right)\right|=\left|z_{n+1}\right| \geqslant \frac{1}{2} r_{n+1} \geqslant \frac{1}{4} M\left(r_{n}\right), n=1,2, \ldots$, so

$$
\varliminf_{n \rightarrow \infty} \frac{\ln \ln \left|f^{n}\left(z_{1}\right)\right|}{\ln N\left(r_{n}\right)} \geqslant \varliminf_{n \rightarrow \infty} \frac{\ln \ln M\left(r_{n}\right)}{\ln N\left(r_{n}\right)} \geqslant \frac{1}{1+2 \delta} .
$$

Combining this with (5.20) gives the required estimate.

By choosing $r_{1}$ to be sufficiently large, we can also ensure that $\left|f^{n}\left(z_{1}\right)\right|>R_{0}(f)$, for $n=0,1,2, \ldots$, and $z_{1} \notin E(f)$. We have already remarked that $z_{1} \in A(f)$ and so, by Lemma 5.2, we deduce that $z_{1}$ satisfies all the conditions of Proposition 1.4.

Acknowledgements. The authors are grateful to Walter Bergweiler for helpful discussions, in particular in relation to the ideas for Theorem 1.2.

\section{References}

1. A. F. BeARdon, Iteration of rational functions (Springer, 1991).

2. W. Bergweiler, 'Iteration of meromorphic functions', Bull. Amer. Math. Soc. 29 (1993) 151-188.

3. W. Bergweiler and A. Hinkkanen, 'On semiconjugation of entire functions', Math. Proc. Cambridge Philos. Soc. 126 (1999) 565-574.

4. P. L. Duren, Univalent functions (Springer, 1983).

5. A. E. Eremenko, 'On the iteration of entire functions', Dynamical systems and ergodic theory, Banach Center Publication 23 (Polish Scientific Publishers, Warsaw, 1989) 339-345.

6. A. E. Eremenko and M. Yu. Lyubich, 'Dynamical properties of some classes of entire functions', Ann. Inst. Fourier (Grenoble), 42 (1992) 989-1020.

7. K. J. FAlCONER, Fractal geometry: mathematical foundations and applications (John Wiley, 1990).

8. W. H. J. Fuchs, 'Asymptotic evaluation of integrals, and Wiman-Valiron theory', Complex analysis and its applications. International Seminar, Trieste, 1975 - Vol. I (International Atomic Energy Agency, Vienna, 1976) 235-283.

9. W. K. HAYMAN, 'The local growth of power series: a survey of the Wiman-Valiron method', Canad. Math. Bull. 17 (1974) 317-358.

10. W. K. Hayman, Subharmonic functions - Vol. 2 (Academic Press, 1989).

11. R. Nevanlinna, Analytic functions (Springer, 1970).

12. P. J. Rippon and G. M. Stallard, 'Iteration of a class of hyperbolic meromorphic functions', Proc. Amer. Math. Soc. 127 (1999) 3251-3258.

13. P. J. Rippon and G. M. Stallard, 'On questions of Fatou and Eremenko', Proc. Amer. Math. Soc. 133 (2005) 1119-1126.

14. G. M. Stallard, 'The Hausdorff dimension of Julia sets of entire functions II', Math. Proc. Cambridge Philos. Soc. 119 (1996) 513-536.

15. G. M. Stallard, 'Dimensions of Julia sets of hyperbolic entire functions', Bull. London Math. Soc. 36 (2004) 263-270.

16. E. C. Titchmarsh, The theory of functions (Oxford University Press, 1939).

17. M. Tsuji, Potential theory in modern function theory (Maruzen, Tokyo, 1959).

18. M. URBAnski, 'Geometry and ergodic theory of conformal non-recurrent dynamics', Ergodic Theory Dynam. Systems 17 (1997) 1449-1476.

19. G. Valiron, Fonctions analytiques (Presses Universitaires de France, Paris, 1954).

\author{
P. J. Rippon and G. M. Stallard \\ Department of Pure Mathematics \\ Open University \\ Walton Hall \\ Milton Keynes MK7 6AA \\ United Kingdom
}

p.j.rippon@open.ac.uk g.m.stallard@open.ac.uk 